\title{
AVALIAÇÃO DE MÉTOdOS DE ESTIMATIVA DE NÚMERO DE HORAS FRIO PARA O SUL E SUDOESTE DO PARANÁ
}

\author{
SANTOS, Diego Felipe dos - dfsantos17@hotmail.com \\ Universidade Federal de Itajubá/ UNIFEI
}

LEITE, Rayan Reis Miranda - rayanmirandda@gmail.com

Universidade Federal de Itajubá/ UNIFEI

MARTINS, Fabrina Bolzan - fabrinabm@gmail.com

Universidade Federal de Itajubá/ UNIFEI

\begin{abstract}
RESUMO: As frutíferas de clima temperado necessitam acumular horas de frio para sair da dormência e iniciar a floração. Essas frutíferas são importantes para o Paraná; que é o quarto estado com maior produção. Por isso, o objetivo desse trabalho é avaliar o desempenho de quatro métodos de estimativa de número de horas de frio (NHF) para as cidades Fernandes Pinheiro, Francisco Beltrão, Guarapuava, Palmas, Pato Branco e Planalto; localizadas na região sul e sudoeste do Paraná relativo ao período de 1980 a 2014. Para selecionar o melhor método de NHF foi realizada a análise de variância, seguida de comparação de médias, pelo teste Tukey $(a=0,05)$, além da correlação $(r)$, raiz do quadrado médio do erro (RQME) e BIAS. Para todas as cidades, o método de Pola e Angelocci foi o mais eficiente em estimar o número de horas de frio para a região estudada, com menores valores de RQME, entre 26,4 a 46,4 horas. O método de Carbone e Schwartz foi o que apresentou os piores resultados em todas as cidades.
\end{abstract}

Palavras- chaves: necessidade de frio, frutíferas de clima temperado, frio de inverno.

CHILLING HOURS PERFORMANCE METHODS FOR THE SOUTH AND SOUTHWEST OF PARANÁ STATE

ABSTRACT: Many temperate fruit require cold temperatures during the dormancy season to initiate flowering and bear fruit. These fruits are important to the Paraná state; which is the fourth most important in the production of this crop. The aim of this study is to evaluate the performance of four chilling hours methods for Fernandes Pinheiro, Francisco Beltrão, Guarapuava, Palmas, Pato Branco and Planalto; located at south and southwest of Paraná, for the period 1980 to 2014. To verify the performance of four methods, the accumulated chilling hours were submitted to analysis of variance followed by Tukey test $(a=0.05)$, Pearson correlation $(r)$, root mean square error (RMSE) and BIAS. The Pola and Angelocci method estimations performed better, with lower RMSE values, 26.4 to 46.4 hours. The Carbone and Schwartz method was the worst chilling hours estimated.

Keywords: chilling requirements, temperate climate fruits, winter chill.

\section{INTRODUÇÃO}

As frutíferas de clima temperado e as espécies florestais criófilas caracterizam-se pela necessidade de um período de dormência no qual há inatividade fisiológica e nenhum crescimento visível (LUEDELING et al., 2012; WREGE et al., 2016). O período de dormência somente é quebrado pela ação benéfica do frio invernal, que deve ser suficiente para satisfazer as exigências fisiológicas dessas plantas (HELDWEIN et al., 1989; LUEDELING et al., 2011). A 
insuficiência de frio invernal causa anomalias fisiológicas, como a queda das gemas frutíferas, atraso, irregularidade ou não ocorrência da brotação e floração, afetando negativamente o desenvolvimento, crescimento e a produtividade (BURIOL et al., 2000; HELDWEIN et al., 2000; LUEDELING et al., 2011). Devido à limitação fisiológica das frutíferas de clima temperado e para garantir o manejo adequado dessas culturas no campo (LUEDELING, 2012), além de determinar as áreas climatologicamente aptas a essas culturas, é imprescindível quantificar a quantidade e a climatologia de frio disponível no local de cultivo.

O frio invernal é quantificado através de unidades e/ou número de horas de frio (NHF) e representa o acúmulo de horas em que a temperatura do ar permanece abaixo de determinada temperatura base $(\mathrm{Tb})$, sendo variável para cada espécie/cultivar (HELDWEIN et al., 1989; LUEDELING, 2012). Normalmente, convenciona-se utilizar a $\mathrm{Tb}=7^{\circ} \mathrm{C}$ como o limite para a superação da dormência em frutíferas mais exigentes em frio (CAMPOY et al., 2011).

O NHF pode ser contabilizado diretamente nos termogramas ou nas estações automáticas, porém é uma atividade extremamente trabalhosa, principalmente quando é necessário realizar correções nesses registros. Além disso, a grande maioria das estações meteorológicas é carente em termógrafos e quando existem, possuem falhas nos registros. Já as estações automáticas além de possuírem falhas, também possuem poucos anos com dados disponíveis, o que inviabiliza a observação climatológica do NHF. Por esses motivos, é necessário o uso de métodos indiretos para a quantificação do NHF (HELDWEIN et al., 1989; CAMPOY et al., 2011; DARBYSHIRE et al., 2013).

Existe uma infinidade de métodos para estimar o NHF, os quais se baseiam em regressões (PEDRO JR. et al., 1979; HELDWEIN et al., 1989; BURIOL et al., 2000; HELDWEIN et al., 2000), condições para unidades de frio (RICHARDSON et al., 1974; ALVARENGA et al., 2002), métodos analíticos (ANGELOCCI et al., 1979; LINVILL, 1990; POLA; ANGELOCCI, 1993), ou modelos dinâmicos (LUEDELING et al., 2009). No entanto, a maioria não tem sentido prático; pois muitos métodos são específicos para os locais em que foram estimados, como os métodos de regressão; ou foram estimados para condições climáticas do hemisfério norte, além de contabilizar mensalmente o NHF para o valor de $\mathrm{Tb}$ entre $0^{\circ}$ e $7,2^{\circ} \mathrm{C}$, sendo impossível estimar considerando outros valores de Tb.

No Brasil, a maioria dos estudos sobre a estimativa do NHF são antigos (ANGELOCCI et al., 1979; PEDRO JR. et al., 1979; HELDWEIN et al., 1989) e defasados, pois não se sabe se esses métodos estimam razoavelmente o NHF para as condições de clima atual, que comprovadamente tem alterações nos campos médios sazonais e de variabilidade em diversos elementos meteorológicos, principalmente na temperatura do ar (TORRES; MARENGO, 2014). Informações do NHF são importantes para a região sul do Brasil, as quais investem no cultivo de frutíferas de clima temperado e espécies florestais criófilas (WREGE et al., 2016). Nesse sentido, destaca-se o Paraná, sendo um dos principais produtores de frutíferas de clima temperado, com destaque para uva, morango e maçã (ANDRADE, 2015), sendo o estado que ocupa a quarta posição nacional (BRUNETTO et al., 2016), atrás somente dos estados do Rio Grande do Sul, Santa Catarina e São Paulo. Portanto, o objetivo desse trabalho 
é avaliar e comparar o desempenho de quatro métodos de estimativa do NHF diário para a região sul e sudoeste do estado do Paraná.

\section{MATERIAL E MÉTODOS}

Foram utilizados dados diários de temperaturas das $21 \mathrm{~h}\left({ }^{\circ} \mathrm{C}\right)$, mínima $\left({ }^{\circ} \mathrm{C}\right)$, máxima $\left({ }^{\circ} \mathrm{C}\right)$ e número de horas de frio abaixo de $7^{\circ} \mathrm{C}$ (h) (NHF observado), disponibilizados pelo Instituto Agronômico do Paraná (IAPAR), para os meses de maio a setembro de 1980 a 2014. Os dados são provenientes das estações meteorológicas convencionais localizadas em Fernandes Pinheiro, Francisco Beltrão, Guarapuava, Palmas, Pato Branco e Planalto (Figura 1). Anos com mais de $5 \%$ de dados faltantes no período de maio a setembro foram excluídos.

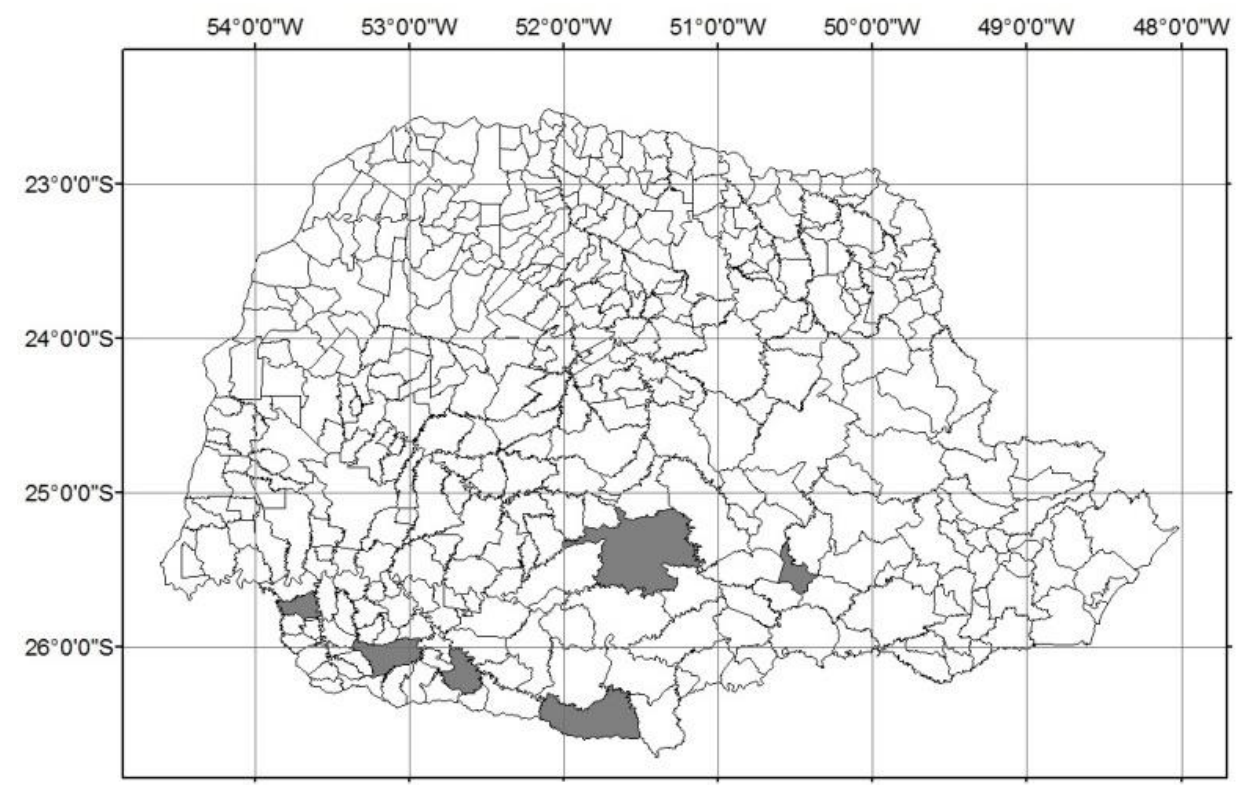

Figura 1 - Localização geográfica das estações meteorológicas que apresentam dados disponíveis para a estimativa do número de horas frio no estado do Paraná.

Os métodos utilizados para a estimativa do NHF diário foram os métodos de Angelocci et al. (1979), Angelocci modificado, Carbone e Schwartz (1993) e o método de Pola e Angelocci (1993). A escolha dos métodos baseou-se em serem métodos analíticos capazes de estimar o NHF para qualquer valor de Tb, além de utilizarem variáveis de fácil medição e maior disponibilidade nas estações.

O método de Angelocci et al. (1979) (NHFa) aproxima a curva diária de temperatura com uma série de segmentos de reta (Figura 2), considerando 7 casos possíveis para o acúmulo diário do NHF. Nesses 7 casos, todas as condições separadas por ";" devem ser atendidas para se obter o NHF. 


$$
\begin{gathered}
\text { Equações } \\
N H F a=\left\{\begin{array}{l}
\frac{9(T b-T m)}{T 21-T m}+\frac{8(T b-T m)}{T M 2-T m} \\
\frac{6(T b-T 21)}{T M 1-T 21}+\frac{8(T b-T m)}{T M 2-T m} \\
\frac{8(T b-T m)}{T M 2-T m}+16 \\
\frac{6(T b-T 21)}{T M 1-T 21}+17 \\
\frac{9(T b-T m)}{T 21-T m}+8 \\
24 \\
0
\end{array}\right.
\end{gathered}
$$

Condições

$\mathrm{Tb}>\mathrm{Tm} ; \mathrm{Tb} \leq \mathrm{T} 21 ; \mathrm{Tb}<\mathrm{TM} 1 ; \mathrm{Tb}<\mathrm{TM} 2$

$\mathrm{Tb}>\mathrm{Tm} ; \mathrm{Tb} \geq \mathrm{T} 21 ; \mathrm{Tb}<\mathrm{TM} 1 ; \mathrm{Tb}<\mathrm{TM}$

Tb >Tm; Tb $\geq T M 1 ; T b \leq T M 2$

$\mathrm{Tb}>\mathrm{Tm} ; \mathrm{Tb} \geq \mathrm{TM} 2 ; \mathrm{Tb} \geq \mathrm{T} 21 ; \mathrm{Tb}<\mathrm{TM}$

$\mathrm{Tb}>\mathrm{Tm} ; \mathrm{Tb} \leq \mathrm{T} 21 ; \mathrm{Tb}<\mathrm{TM} 1 ; \mathrm{Tb}>\mathrm{TM} 2$

$\mathrm{Tb} \geq \mathrm{TM} 1 ; \mathrm{Tb} \geq \mathrm{TM} 2$

$\mathrm{Tb} \leq \mathrm{Tm}$

Em que: NHFa é o número de horas de frio diário ( $h$ ) baseado no método de Angelocci; Tb=temperatura base da frutífera de clima temperado, neste caso considerou-se $70 \quad \mathrm{C} \quad\left({ }^{\circ} \mathrm{C}\right) ; \quad \mathrm{Tm}=$ temperatura mínima do ar $\left({ }^{\circ} \mathrm{C}\right)$; $\mathrm{TM} 1=$ temperatura máxima do ar do dia anterior $\left({ }^{\circ} \mathrm{C}\right) ; \mathrm{TM} 2=$ temperatura máxima do ar do dia em questão $\left({ }^{\circ} \mathrm{C}\right) ; \mathrm{T} 21=$ temperatura do ar às $21 \mathrm{~h}\left({ }^{\circ} \mathrm{C}\right)$, entre as temperaturas máximas ocorridas em dois dias consecutivos.

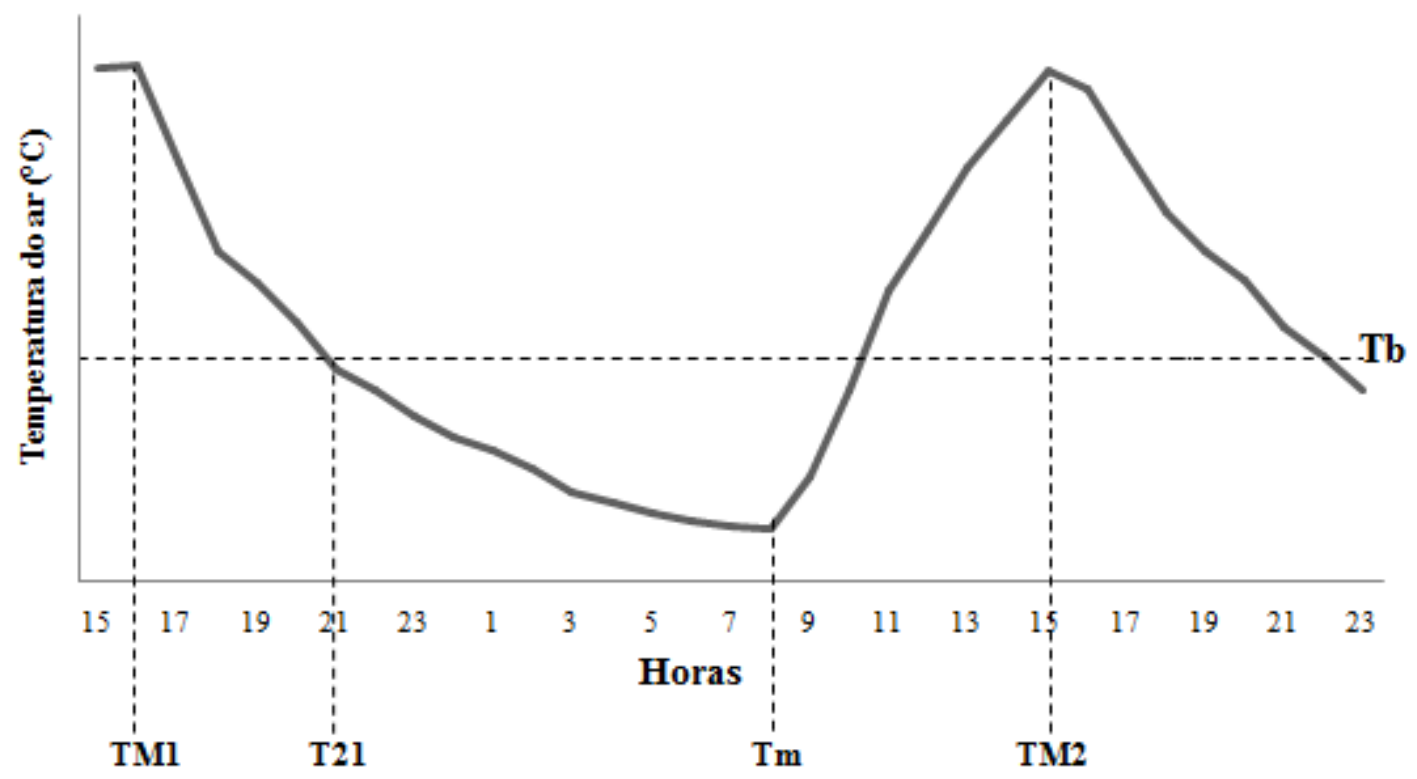

Figura 2 - Representação gráfica da curva diária da temperatura do ar, do cômputo do número de horas frio e das temperaturas utilizadas no método de Angelocci et al. (1979).

No método de Angelocci et al. (1979) não é especificado se a T21 é referente a do dia ou a do dia anterior. Por isso, no método Angelocci modificado (NHFam) utilizou-se a T21 ocorrida no dia em questão (Figura 3), considerando os mesmos 7 casos do método tradicional (NHFa). 


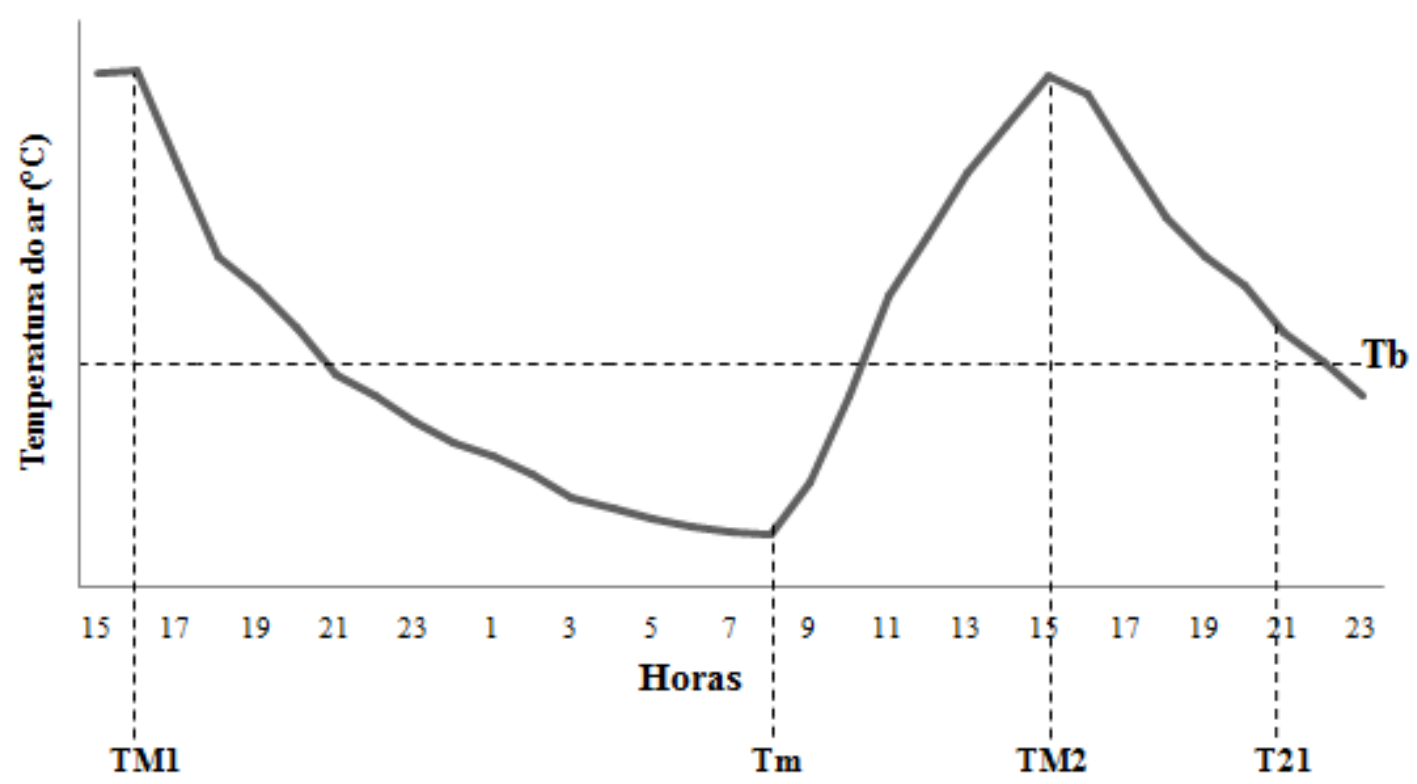

Figura 3 - Representação gráfica da curva diária da temperatura do ar, do cômputo do número de horas frio e das temperaturas utilizadas no método de Angelocci modificado.

O método de Pola e Angelocci (1993) (NHFpa) baseia-se na variação ondulatória média da temperatura do ar, onde a temperatura mínima (Tm) ocorre próxima às $7 \mathrm{~h}$ e as temperaturas máximas (TM1 e TM2) ocorrem próximas às $15 \mathrm{~h}$ (Figura 4). Dessa forma, é possível aproximar-se a onda de temperatura diária por duas funções senoidais, uma de resfriamento (equação 2) (aplicada no intervalo de tempo da TM1 a Tm) e uma de aquecimento (equação 3) (aplicada no intervalo de tempo da Tm a TM2). Considerando um período de 24 horas compreendido entre as duas temperaturas máximas, temos que a TM1 ocorre no tempo inicial $\left(\mathrm{t}_{\mathrm{M} 1}=0 \mathrm{~h}\right)$, a Tm ocorre no tempo decorrido das $15 \mathrm{~h}$ do dia anterior até as $7 \mathrm{~h}$ do dia $(\mathrm{tm}=16 \mathrm{~h})$ e a TM2 ocorre no tempo decorrido das $15 \mathrm{~h}$ do dia anterior até as $15 \mathrm{~h}$ do dia em questão (tM2=24h). As funções de resfriamento e aquecimento devem ser calculadas de forma horária, sendo possível detectar os momentos exatos, no tempo, em que as temperaturas se tornam iguais à $\mathrm{Tb}$.

A temperatura que ocorre durante o resfriamento diário é dada por:

$\operatorname{Tr}(\mathrm{t})=\operatorname{TM} 1+(\mathrm{Tm}-\mathrm{TM} 1) \cdot \sin \left|\frac{\pi}{2} \cdot \frac{\left(t-t_{M 1}\right)}{\left(t_{m}-t_{M 1}\right)}\right|$

Em que: $\operatorname{Tr}(\mathrm{t})$ é a temperatura estimada no tempo $t$ durante o resfriamento diário $\left({ }^{\circ} \mathrm{C}\right)$ e t é o tempo $(\mathrm{h})$ que varia de $\mathrm{tM} 1 \mathrm{a}$ tm, isto é, $0 \leq \mathrm{t} \leq 16$.

A temperatura que ocorre durante o aquecimento diário é dada por:

$\mathrm{Ta}(\mathrm{t})=\mathrm{Tm}+(\mathrm{TM} 2-\mathrm{Tm}) \cdot \sin \left|\frac{\pi}{2} \cdot \frac{\left(t-t_{m}\right)}{\left(t_{M 2}-t_{m}\right)}\right|$

Em que: Ta ( $\mathrm{t}$ ) é a temperatura estimada no tempo $\mathrm{t}$ durante o aquecimento diário $\left({ }^{\circ} \mathrm{C}\right)$ e t é o tempo $(\mathrm{h})$ que varia de tm a tM2, isto é, $16 \leq \mathrm{t} \leq 24$.

Simbolizando por "ta" o tempo $t$ em que $\operatorname{Tr}(\mathrm{t})$ decai ou iguala-se a Tb, isto é, $\operatorname{Tr}(\mathrm{ta}) \leq \mathrm{Tb}$, e por "tz" o tempo $\mathrm{t}$ em que $\mathrm{Ta}(\mathrm{t})$ supera ou iguala-se a $\mathrm{Tb}$, isto é, $\mathrm{Ta}(\mathrm{tz}) \geq \mathrm{Tb}$ (Figura 4 ), obtemos 5 casos possíveis para estimativa de 
NHFpa. No primeiro caso, como a Tb é menor que as temperaturas máximas e mínima, temos que o NHFpa é nulo, isto é, durante 0 horas do dia a temperatura do ar ficou abaixo da Tb. No segundo caso, como a Tb é maior que as temperaturas máximas, temos que o NHFpa é acumulado durante todo o dia, isto é, a temperatura do ar ficou abaixo da Tb durante 24 horas. Nos demais casos temos uma relação entre os tempos $t_{M 1}$, $t_{M 2}$, ta e tz. Nesses 5 casos, todas as condições separadas por ";" devem ser atendidas para se obter o NHFpa.

NHFpa $= \begin{cases}0, & \mathrm{TM} 1>\mathrm{Tb} ; \mathrm{TM} 2>\mathrm{Tb} ; \mathrm{Tm}>\mathrm{Tb} \\ 24, & \mathrm{TM} 1<\mathrm{Tb} ; \mathrm{TM} 2<\mathrm{Tb} \\ \mathrm{tz}-\mathrm{ta}, & \mathrm{TM} 1>\mathrm{Tb} ; \mathrm{TM} 2<\mathrm{Tb} ; \mathrm{Tm}<\mathrm{Tb} \\ \mathrm{tz}-\mathrm{t}_{\mathrm{M} 1}, & \mathrm{TM} 1 \leq \mathrm{Tb} ; \mathrm{TM} 2>\mathrm{Tb} ; \mathrm{Tm}<\mathrm{Tb} \\ \mathrm{t}_{\mathrm{M} 2}-\mathrm{ta}, & \mathrm{TM} 1>\mathrm{Tb} ; \mathrm{TM} 2 \leq \mathrm{Tb}\end{cases}$

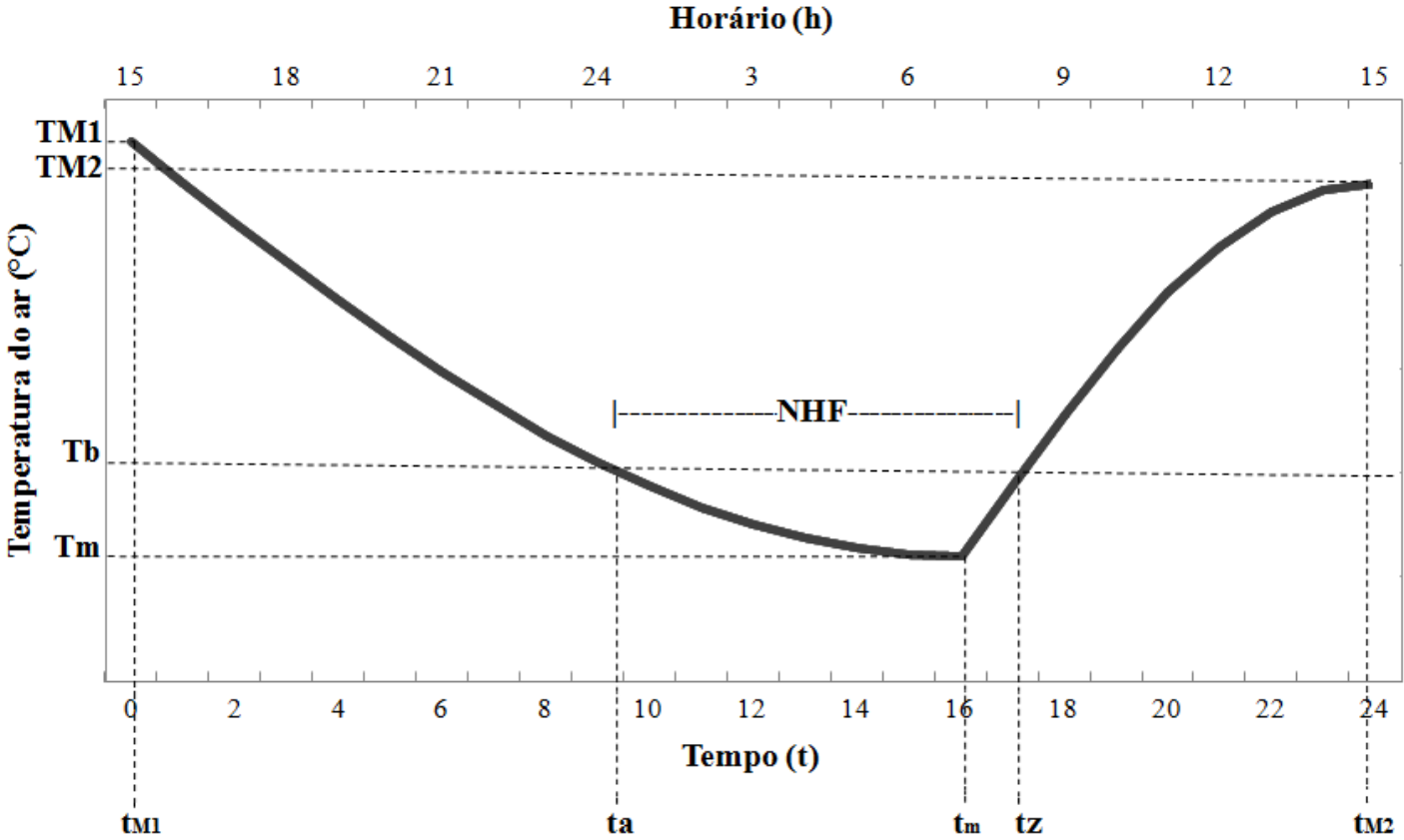

Figura 4 - Representação gráfica da curva diária da temperatura do ar, do cômputo do número de horas frio e das temperaturas utilizadas no método de Pola e Angelocci (1993).

O método de Carbone e Schwartz (1993) (NHFcs) faz adaptações ao método de Linvill (1990), com melhorias e correções. Esse método, assim como o NHFpa baseia-se na curva de temperatura diária (Figura 5), determinando o NHF para os períodos anterior e posterior ao pôr do sol.

$$
\begin{aligned}
& \delta=23,45^{\circ} * \sin \left[\frac{360^{\circ} *(284+\mathrm{NDA})}{365}\right] \\
& \mathrm{DL}=0,1333 * \cos ^{-1}[-1 *(\tan \emptyset * \tan \delta)] \\
& \mathrm{Ts}=(\mathrm{TM} 2-\mathrm{Tm}) * \sin \left[\frac{\pi * D L}{D L+4}\right]+\mathrm{Tm} \\
& \mathrm{CH} 1=\left(\frac{\mathrm{DL}+4}{\pi}\right) * \sin ^{-1}\left(\frac{\mathrm{Tb}-\mathrm{Tm}}{\mathrm{TM} 2-\mathrm{Tm}}\right)
\end{aligned}
$$


$\mathrm{CH} 2=(24-\mathrm{DL})+\exp ^{\left[\frac{\mathrm{Ts}-\mathrm{Tb}}{\mathrm{Ts}-\mathrm{Tm} 2}\right]}$

$\mathrm{NHFCS}= \begin{cases}\mathrm{CH} 1+\mathrm{CH} 2, & \mathrm{CH} 1+\mathrm{CH} 2 \geq 0 \\ 0, & \mathrm{CH} 1+\mathrm{CH} 2<0\end{cases}$

Em que: $\delta$ é a declinação solar (graus e décimos); NDA é o número do dia no ano ou dia juliano; DL é a duração de luz no dia ou fotoperíodo (h); "Ø" é a latitude (graus e décimos); Ts é a temperatura no horário do pôr do sol $\left({ }^{\circ} \mathrm{C}\right)$; $\mathrm{CH} 1$ é o NHF após o nascer do sol (h); $\mathrm{CH} 2$ é o NHF após o pôr do sol (h); Tm2 é a temperatura mínima do ar do dia seguinte $\left({ }^{\circ} \mathrm{C}\right)$.

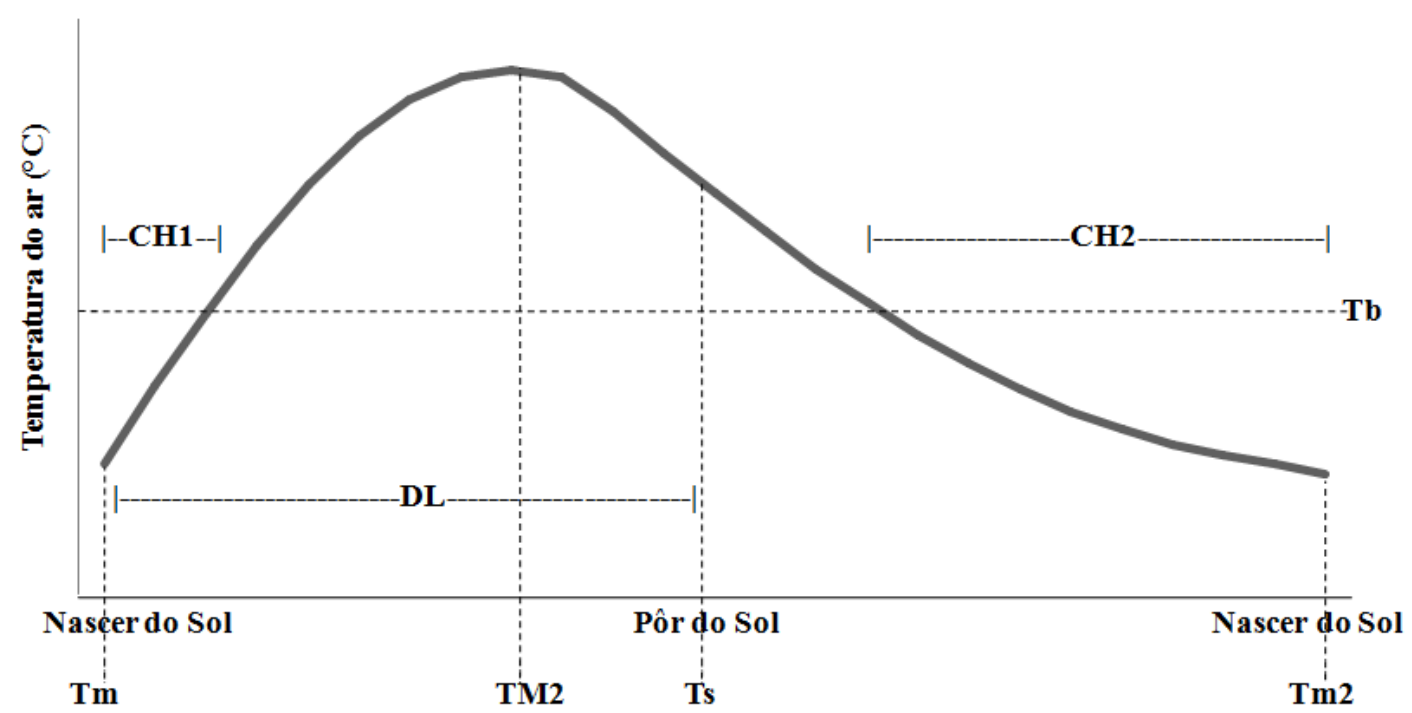

Figura 5 - Representação gráfica da curva diária da temperatura do ar, do cômputo do número de horas frio e das temperaturas utilizadas no método de Carbone e Schwartz (1993).

Em todos os métodos, os valores de NHF foram estimados diariamente, para o período compreendido entre maio a setembro, e posteriormente foram acumulados para valores anuais (NHFanual).

Para avaliar e comparar o desempenho dos métodos de estimativa do NHF, procedeu-se a análise de variância (ANOVA) para a fonte de variação métodos (NHFa, NHFam, NHFpa, NHFcs e NHF observado), seguida da comparação de médias pelo teste Tukey $(a=0,05)$, considerando o NHFanual. Os testes foram feitos no software SISVAR versão 5.3 (FERREIRA, 2011).

Além disso, foram realizadas as estatísticas raiz do quadrado médio do erro (RQME), BIAS e correlação de Pearson (r). O RQME expressa o erro médio do método de estimativa, e quanto menor o valor, melhor é o método (CESARACCIO et al., 2004). O BIAS expressa a tendência do método, e leva em consideração o desvio médio do NHFanual estimados em relação ao NHFanual observados. Quanto menor o valor de BIAS menos tendencioso e melhor é o método de NHF (LEITE; ANDRADE, 2002). E r determina o grau de associação entre duas ou mais variáveis, sendo que quanto mais próximo de 1 (positivo ou negativo), maior a relação entre valores observados e estimados e melhor é o método de estimativa de NHF (MARTINS et al., 2014): 


$$
\begin{aligned}
& R Q M E=\left[\sum_{i=1}^{n}(E i-O i)^{2} / n\right]^{0,5} \\
& B I A S=\sum_{i=1}^{n} E i-\sum_{i=1}^{n} O i / \sum_{i=1}^{n} O i \\
& r=\sum_{i=1}^{n}(O i-\bar{O})(E i-\bar{E}) /\left\{\left[\sum_{i=1}^{n}(O i-\bar{O})^{2}\right]\left[\sum_{i=1}^{n}(E i-\bar{E})^{2}\right]\right\}^{0,5}
\end{aligned}
$$

Em que: Ei são os valores de NHF estimados por cada método; Oi são os valores de NHF observados; $n$ é o número de observações; $\overline{\mathrm{E}}=$ média do NHF estimados pelos métodos e $\overline{0}=$ média do NHF observados.

\section{RESULTADOS E DISCUSSÃO}

Os maiores valores observados de NHF anuais foram registrados em Palmas, enquanto os menores valores foram registrados em Planalto (Figura 6 e Tabela 1). Esse padrão é esperado, tendo em vista que Planalto é localizado em uma região de baixas altitudes, formada por vales e rios; desfavorecendo o acúmulo de NHF, enquanto Palmas localiza-se em regiões de maiores altitudes e latitude, que favorecem a ocorrência de temperaturas amenas e consequentemente maior acúmulo de NHF (WREGE et al., 2016).

Além disso, verifica-se tanto nas estimativas quanto nos dados observados de NHFanual, que as regiões sul e sudoeste do Paraná apresentam desvio padrão alto e consequentemente uma variabilidade anual alta (Figura 6). Nos dados observados, o desvio padrão do NHF anual varia de 51,72; em Planalto, a 111,36; em Palmas. Essa variabilidade é inerente a variação anual da temperatura do ar, além da atuação dos sistemas meteorológicos predominantes na região, principalmente a entrada de frentes frias, as quais adentram no sul do estado e causam queda brusca nas temperaturas (COSTA; ANDRADE, 2015; FRITZSONS et al., 2008). No entanto, a variabilidade interanual do somatório de frio é prejudicial ao estabelecimento das frutíferas de clima temperado, dificultando a escolha das cultivares para cada região; nos anos de menor acúmulo de frio a brotação e o florescimento podem ser prejudicados e, consequentemente, pode ocorrer redução na produtividade (TANASIJEVIC et al., 2014) e dificuldade de escolha das cultivares com melhor adaptação (MORAIS; CARBONIERI, 2015; MAULIÓN et al., 2014). 


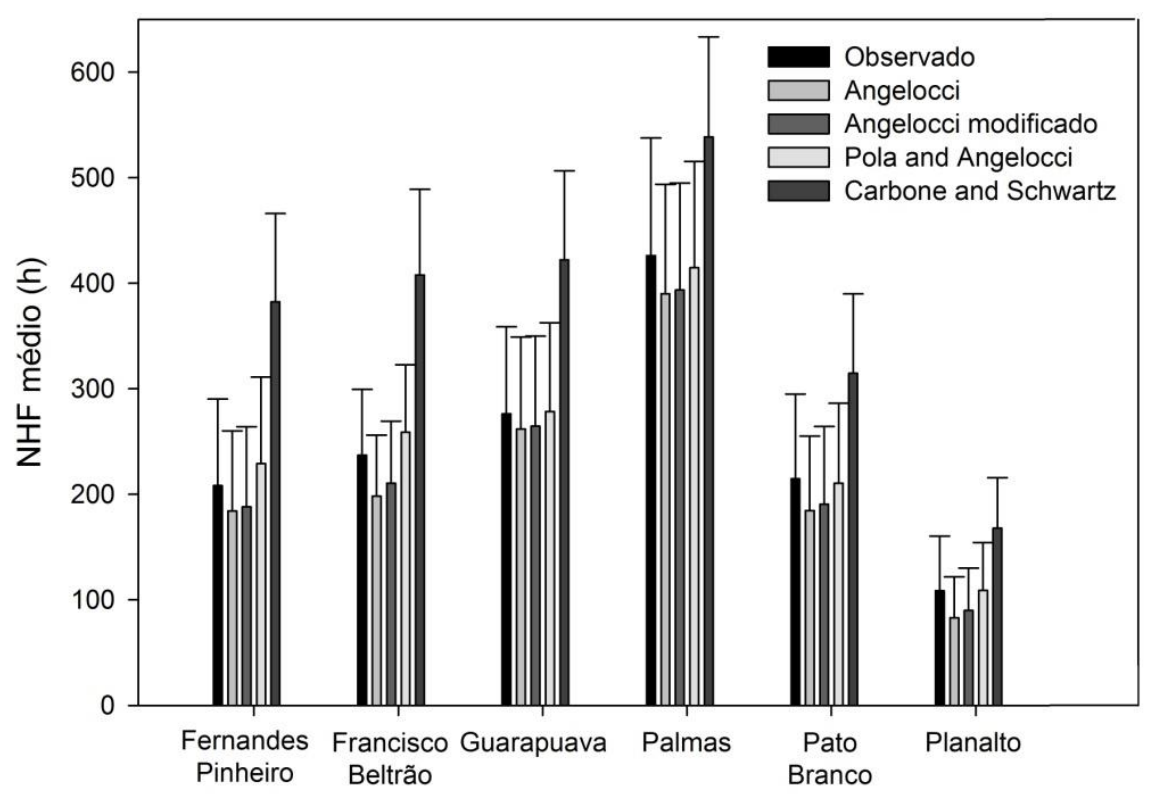

Figura 6 - Média e desvio padrão do Número de Horas Frio anual no período de 1980 a 2014, para as cidades de Fernandes Pinheiro, Francisco Beltrão, Guarapuava, Palmas, Pato Branco e Planalto.

Pela comparação de médias (Tabela 1), os métodos NHFa, NHFam e NHFpa não apresentarem diferença significativa em relação ao NHF observado, contrário do método de NHFcs. No entanto, os métodos NHFa e NHFam subestimaram o NHF, enquanto o NHFcs superestimou o NHF em torno de 127,09 horas. O método NHFpa mais se aproximou do NHF observado, com uma diferença de apenas 4,87 horas em média por ano, o que pode ser considerado desprezível, representando um erro de acúmulo diário de NHF inferior a 0,0333 horas, cerca de 2 minutos.

Tabela 1 - Comparação de médias e desvio padrão para os métodos de estimativa do Número de Horas Frio para seis cidades do Paraná (PR), no período de 1980 a 2014.

\begin{tabular}{lcccccc}
\hline Cidades & $\begin{array}{l}\text { Desvio } \\
\text { Padrão }\end{array}$ & Observado & Angelocci & $\begin{array}{l}\text { Angelocci } \\
\text { Modificado }\end{array}$ & $\begin{array}{l}\text { Pola e } \\
\text { Angelocci }\end{array}$ & $\begin{array}{l}\text { Carbone } \\
\text { e } \\
\text { Schwartz }\end{array}$ \\
\hline Fernandes P. & 14,14 & $208,31 \mathrm{a}$ & $188,11 \mathrm{a}$ & $184,23 \mathrm{a}$ & $229,18 \mathrm{a}$ & $382,43 \mathrm{~b}$ \\
Francisco B. & 11,07 & $237,20 \mathrm{ab}$ & $210,62 \mathrm{a}$ & $198,36 \mathrm{a}$ & $258,94 \mathrm{~b}$ & $407,96 \mathrm{c}$ \\
Guarapuava & 14,37 & $276,34 \mathrm{a}$ & $264,67 \mathrm{a}$ & $261,85 \mathrm{a}$ & $278,34 \mathrm{a}$ & $422,17 \mathrm{~b}$ \\
Palmas & 18,47 & $426,26 \mathrm{a}$ & $393,67 \mathrm{a}$ & $390,11 \mathrm{a}$ & $414,74 \mathrm{a}$ & $538,66 \mathrm{~b}$ \\
Pato Branco & 15,08 & $214,68 \mathrm{a}$ & $190,73 \mathrm{a}$ & $184,65 \mathrm{a}$ & $210,48 \mathrm{a}$ & $314,74 \mathrm{~b}$ \\
Planalto & 7,61 & $108,66 \mathrm{a}$ & $89,98 \mathrm{a}$ & $83,15 \mathrm{a}$ & $109,00 \mathrm{a}$ & $168,01 \mathrm{~b}$ \\
\hline
\end{tabular}

Médias seguidas pela mesma letra minúscula na linha, não diferem pelo teste Tukey a $5 \%$.

Analisando as demais estatísticas (Tabela 2), observa-se para todas as cidades, exceto Palmas, que os métodos NHFa, NHFam e NHFpa, apresentam elevados valores de correlação $(\geq 0,85)$ e baixos valores de RQME $(\leq 46,53 \mathrm{~h})$ e de BIAS $(-0,23)$, sugerindo que os três métodos são coerentes em estimar o NHF, corroborando os resultados da Tabela 1. Já o NHFcs apresentou valores de correlação inferior aos demais métodos $(\leq 0,76)$ e erro elevado, em torno de 150 horas, sendo considerado um método inviável para estimar o NHF. 
De maneira geral, verifica-se proximidade entre os valores de NHF estimado pelos métodos NHFa, NHFam e NHFpa em relação ao NHF observado (Tabelas 1 e 2). No entanto, a proximidade é maior quando o NHF observado anual é menor (em torno de 200h), e decai quando o NHF é maior (Figura 7). Heldwein et al. (1989) também observaram melhor estimativa do NHF, considerando métodos analíticos e de regressão, para os meses menos frios em Santa Maria (RS). Isso ocorreu possivelmente em função da menor variação do momento de ocorrência das temperaturas extremas (TM e Tm) em dias que apresentavam marcha diária da temperatura similar ao padrão, considerando dias sem nebulosidade. A presença de nuvens afeta o balanço de energia; desta forma, durante a noite contribui para a diminuição da quantidade de radiação de onda longa perdida para o espaço, aumentando a temperatura mínima, e durante o dia contribui para que uma quantidade menor de radiação de onda curta alcance a superfície, diminuindo a temperatura máxima (SILVA; GUETTER, 2003). Já Botelho et al. (2006) testando métodos de unidades de frio (UF) e de número de horas de frio para o Paraná, verificaram que em regiões com invernos mais amenos, ocorre maior diferença entre NHF $\leq 7,20 \mathrm{C}$ e o número de UF. Isso ocorre principalmente em decorrência do método de UF ponderar diferentes faixas de temperatura; resultando em menores acúmulos de unidades de frio em dias com temperaturas altas, mesmo no período invernal (CARDOSO et al., 2015). Salienta-se que o método de UF é bastante utilizado nos EUA (LINVILL, 1990; LUEDELING, 2012) e pouco utilizado no Brasil (ALVARENGA et al., 2002), principalmente por considerar unidades de frio ponderadas por faixas de temperatura do ar.

Tabela 2. Estatísticas utilizadas na avaliação do desempenho dos métodos de estimativa de Número de Horas Frio para seis cidades do Paraná (PR), no período de 1980 a 2014.

\begin{tabular}{|c|c|c|c|c|c|c|c|c|c|c|c|}
\hline \multirow{2}{*}{ Cidades } & \multicolumn{3}{|c|}{ Angelocci } & \multicolumn{3}{|c|}{ Angelocci Modificado } & \multicolumn{2}{|c|}{ Pola e Angelocci } & \multicolumn{3}{|c|}{ Carbone e Schwartz } \\
\hline & RQME & BIAS & $r$ & RQME & BIAS & 1 & RQME BIAS & 1 & RQME & BIAS & $\mathrm{r}$ \\
\hline ernandes P & 3 & & 0 & & & 0,95 & 351601 & 0,94 & & 0,72 &, 75 \\
\hline Franc & 30 & -0 & 0,97 & 4 & $-0,16$ & 0,96 & 60,0 & 0,97 & 157,1 & 0,53 & 0,75 \\
\hline & 3 & -0 & 0,93 & & -0 , & 0,92 & 390, & 0,93 & & 0,26 & 0,69 \\
\hline alm & 5 & $-0,08$ & 0,9 & 62 & $-0,08$ & 0,88 & $46,43-0,03$ & 0,91 & 117,83 & 0,47 & 0,67 \\
\hline & & & $U_{t}$ & & & 0,89 & $2-0,02$ & 0,89 & & 0,55 & 0,76 \\
\hline Id & 33,11 & -0 & 0,85 & 37,24 & $-0,23$ & 0,85 & 26,38 & 0,86 & 183,26 & 0,84 & 0,75 \\
\hline
\end{tabular}

Analisando separadamente os métodos de NHFa, NHFam, verifica-se que ambos estimam valores semelhantes, apesar de considerar diferentes dados de T21 (Figuras 2 e 3), e subestimam o NHF em todas as cidades (Tabela 1 e Figura 7A, B), mesma situação observada por Estefanel et al. (1991) e Botelho et al. (2006). Segundo Angelocci et al. (1979), o método NHFa tende a subestimar o NHF quando utilizado Tb's baixas, como $7^{\circ} \mathrm{C}$. Isso ocorre, pois, as relações trigonométricas do método não representam bem a temperatura do ar no período da tarde, sendo difícil estimar as horas de frio que ocorrem no período das 12 às 24h. O método de NHFpa apresentou subestimativa nas cidades de Palmas e Pato Branco e pequena superestimativa nas demais cidades (Figura 7C), no entanto esses valores foram muito baixos variando o BIAS de 0 a 0,1 (Tabela 2). Inclusive, para as cidades de Guarapuava, Pato Branco e Planalto, os valores do NHFpa foram muito semelhantes ao NHF observado. Já o método de NHFcs superestimou o NHF em todas as cidades (Figura 7D). 
Assim, o NHFa, NHFam e NHFpa podem ser utilizados para a estimativa de NHF. No entanto, em quase todas as cidades, observou-se melhor desempenho do método de NHFpa em relação aos demais. Dessa forma, deve ser dada preferência ao método de NHFpa para a estimativa do NHF para as cidades estudadas, podendo ser utilizado em outras localidades da região, garantindo a quantificação adequada da disponibilidade do NHF. Ressalta que o NHF é uma variável utilizada no processo de seleção de zonas climáticas aptas a introdução de frutíferas de clima temperado (LUEDELING, 2012; WREGE et al., 2016), ajustes do zoneamento agrícola (HELDWEIN et al., 1989) considerando o clima presente e futuro (LUEDELING et al., 2011) e avaliação do acúmulo diário, mensal e anual de NHF, o qual serve para aplicar o manejo adequado dessas culturas no campo (LUEDELING, 2012).

O método de NHFpa possuiu a vantagem de ser capaz de refazer a curva diária de temperatura do ar para qualquer local que disponha de dados de Tm e TM, os quais são facilmente obtidos em estações meteorológicas automáticas e convencionais, inclusive as de $3^{\text {a }}$ classe (WMO, 2008). As funções de resfriamento e aquecimento permitem detectar os momentos exatos em que a temperatura do ar é igual à Tb, facilitando assim, o cômputo do NHF simultaneamente para qualquer valor de $\mathrm{Tb}$, além de ser um método computacionalmente simples de ser implementado. Concomitantemente, o NHFpa estima com confiabilidade o NHF para as condições de clima atual (Tabela 2), devendo ser o método utilizado em projeções do NHF considerando cenários climáticos futuros, permitindo avaliar os possíveis impactos das mudanças climáticas no acúmulo de frio. Informações dessa natureza permitem contribuir com atualização dos zoneamentos de aptidão climática para as frutíferas de clima temperado e as espécies florestais criófilas, em estudos de riscos climáticos associados à introdução e cultivo desses tipos de culturas (LUEDELING, 2012; WREGE et al., 2016), além de ser crucial para a escolha das cultivares mais apropriadas para o local de cultivo (LUEDELING et al., 2009). 
A
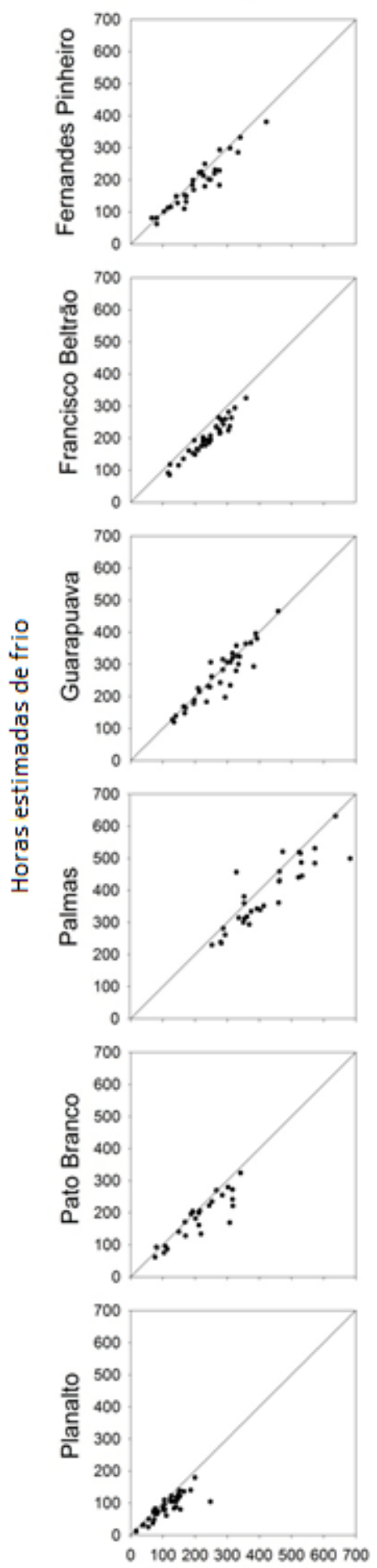

C
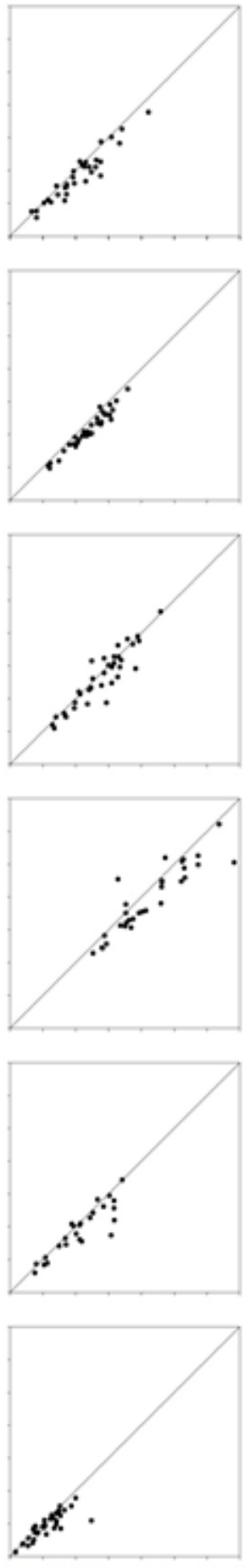

o 100200300400500600700
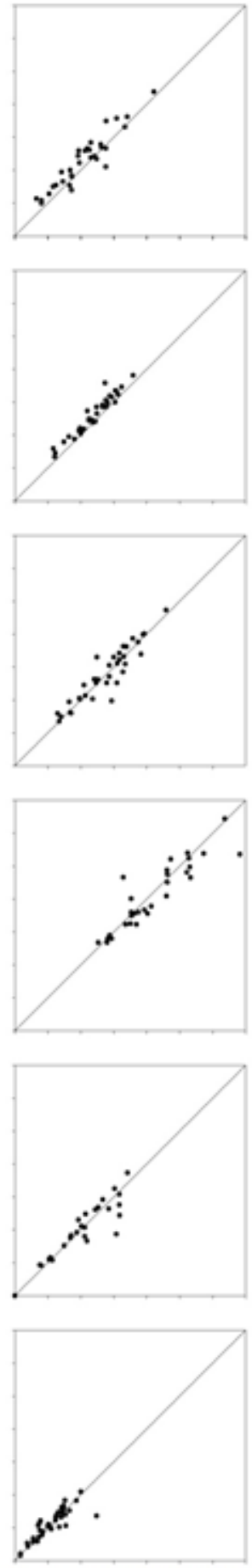

( ) 100200300400500600700
D
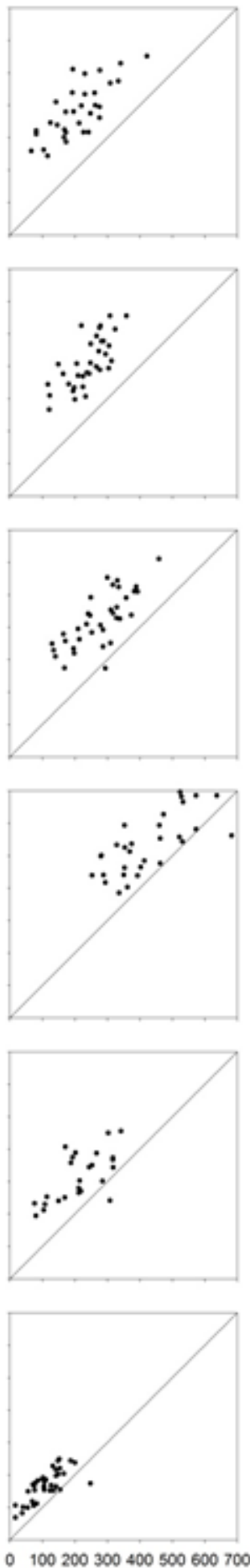

Horas observadas de frio

Figura 7 - Relação entre o número de horas de frio anual observado e o estimado pelos métodos de Angelocci et al. (1979) (painel A), Angelocci modificado (painel B), Pola e Angelocci (1993) (painel C) e Carbone e Schwartz (1993) (painel D). 


\section{CONCLUSÕES}

Dentre os métodos de estimativa do Número de Horas de frio avaliados, o que melhor representa o NHF da região sul e sudoeste do Paraná é o método de Pola e Angelocci (1993), o qual considera as temperaturas máximas e mínimas do ar para recriar a curva de temperatura diária e estabelecer o período no qual a temperatura do ar ficou abaixo de determinado limiar.

\section{AGRADECIMENTOS}

Os autores agradecem ao IAPAR pela disponibilidade dos dados.

\section{REFERÊNCIAS BIBLIOGRÁFICAS}

ALVARENGA, A. A.; ABRAHÃO, E.; RAMOS, J. D.; CHALFUN, N. N. J. Levantamento climático das quantidades de unidades de frio na região de Lavras MG. Ciência e Agrotecnologia, v. 26, n. 6, p. 1344-1347, 2002.

ANDRADE, P. F. S. Fruticultura. Curitiba: SEAB/DERAL, 2015.

ANGELOCCI, L. R.; CAMARGO M. G. P.; PEDRO JR., M. J.; ORTOLANI, A. A.; ALFONSI, R. R. Estimativa do total de horas frias abaixo de determinada temperatura-base através das medidas diárias de temperatura do ar. Revista Científica de Agronomia, v. 38, n. 4, p. 27-36, 1979. http://dx.doi.org/10.1590/S0006-87051979000100004

BOTELHO, R. V.; AYUB, R. A.; MÜLLER, M. M. L. Somatória de horas de frio e de unidades de frio em diferentes regiões do estado do Paraná. Scientia Agraria, v. 7, p. 89-96, 2006. Disponível em: <https://dialnet.unirioja.es/servlet/articulo?codigo=2909639>. Acesso em: 10/10/2016.

BRUNETTO, G.; ROZANE, D. E.; MELO, G. W. B.; ZALAMENA, J.; GIROTTO, E.; LOURENZI, C.; COUTO, R. R.; TIECHER, T.; KAMINSKI, J. Manejo da fertilidade de solos em pomares de frutíferas de clima temperado. In: TIECHER, T. (Org.). Manejo e conservação do solo e da água em pequenas propriedades rurais no Sul do Brasil: práticas alternativas de manejo visando à conservação do solo e da água. Porto Alegre: UFRGS, 2016. p. 141-158.

BURIOL, G. A., ESTENFANEL, V., HELDWEIN, A. B., PRESTES, S. D., OliVEIRA, H. T. de; DIDONÉ, M. A.Disponibilidade de horas de frio na região central do Rio Grande do Sul: 2 - Distribuição Geográfica. Ciência Rural, v. 30, n. 5, p. 755759, 2000. http://dx.doi.org/10.1590/S0103-84782000000500002

CAMPOY, J. A.; RUIZ, D.; EGEA, J. Dormancy in temperate fruit trees in a global warming context: A review. Scientia Horticulturae, v. 130, p. 357-372, 2011. http://dx.doi.org/10.1016/j.scienta.2011.07.011

CARBONE, G. J.; SCHWARTZ, M. D. Potential impact of winter temperature increases on South Carolina peach production. Climate Research, v. 2, p. 225233, 1993. Disponível em: <http://www.intres.com/articles/cr/2/c002p225.pdf>. Acesso em: 07/10/2016.

CARDOSO, L. S.; BERGAMASCHI, H.; BOSCO, L. C.; PAULA, V. A.; NACHTIGAL, G. R. Unidades de frio para macieiras na região de Vacaria - RS, Brasil. Revista 
Brasileira de Fruticultura, v. 37, p. 289-295, 2015. http://dx.doi.org/10.1590/0100-2945-136/14

CESARACCIO, C.; SPANO, D.; SNYDER, R. L.; DUCE, P. Chilling and forcing model to predict bud-burst of crop and forest species. Agriculture and Forest Meteorological, v. 126, p. 2004.http://dx.doi.org/10.1016/j.agrformet.2004.03.002

COSTA, C.; ANDRADE, A. R. Gênese e evolução das temperaturas mínimas absolutas na região centro-sul do Paraná: a influência da dinâmica atmosférica e do relevo local. Boletim Goiano de Geografia, v. 35, n. 3, p. 452-471, 2015. https://doi.org/10.5216/bgg.v35i3.38836

DARBYSHIRE, R.; WEBB, L.; GOODWIN, I.; BARLOW, E. W. R. Impact of future warming on winter chilling in Australia. International Journal of Biometeorology, v. 57, n. 3, p. 355-366, 2013. http://dx.doi.org/10.1007/s00484-012-0558-2

ESTEFANEL, V.; BURIOL, G. A.; SCHNEIDER, F. M.; HELDWEIN, A. B. Ocorrência e duração das temperaturas mínimas diárias do ar prejudiciais à fecundação das flores do arroz em Santa Maria, RS. II - estimativa do número diário de horas com temperaturas baixas. Ciência Rural, v. 21, p. 305-313, 1991. http://dx.doi.org/10.1590/S0103-84781991000300001

FERREIRA, D. F. Sisvar: a computer statistical analysis system. Ciência e Agrotecnologia, v.35, n.6, p.1039-1042, 2011. http://dx.doi.org/10.1590/S1413-70542011000600001

FRITZSONS, E.; MONTOVANI, L. E.; AGUIAR, A. V. Relação entre altitude e temperatura: uma contribuição ao zoneamento climático no estado do Paraná. Revista de estudos ambientais, v.10, n. 1, p. 49-64, 2008.

HELDWEIN, A. B.; ANGELOCCI, L. R.; ESTEFANEL, V.; SCHNEIDER, F. M.; BURIOL, G. A. Avaliação de modelos de estimativa de horas de frio para Santa Maria, RS. Revista Centro de Ciências Rurais, v. 19, p. 45-92, 1989. Disponível em: <http://coral.ufsm.br/revistaccr/index.php/RCCCR/article/view/691/688>. Acesso em: 07/10/2016.

HELDWEIN, A. B.; SCHNEIDER, F. M.; BURIOL, G. A.; ESTEFANEL, V.; PRESTES, S. D. Disponibilidade de horas de frio na região central do Rio Grande do Sul: 1 - Ocorrência de valores acumulados para diferentes níveis de probabilidade. Ciência Rural, v. 30, n. 5, p. 747-754, 2000. http://dx.doi.org/10.1590/S010384782000000500001

LINVILL, D. E. Calculating chilling hours and chill units from daily maximum and minimum temperature observations. HortScience, v. 25, p. 14-16, 1990. Disponível em: <http://hortsci.ashspublications.org/content/25/1/14.full.pdf>. Acesso em: 03/10/2016.

LEITE, H. G.; ANDRADE, V. C. L. Um método para condução de inventários florestais sem o uso de equações volumétricas. Revista Árvore, v.26, n.3, p.321-328, 2002. http://dx.doi.org/10.1590/S0100-67622002000300007

LUEDELING, E. Climate change impacts on winter chill for temperate fruit and nut production: A review. Scientia Horticulturae, v. 144, p. 218-229, 2012.http://dx.doi.org/10.1016/j.scienta.2012.07.011 
LUEDELING, E.; GIRVETZ, E. H.; SEMENOV, M. A.; BROWN, P. H. Climate Change Affects Winter Chill for Temperate Fruit and Nut Trees. PLOS ONE, v. 6, n. 5, p. 20155, 2011.http://dx.doi.org/10.1371/journal.pone.0020155

LUEDELING, E.; ZHANG, M.; LUEDELING, V.; GIRVETZ, E. H. Sensitivity of winter chill models for fruit and nut trees to climatic changes expected in California's Central Valley. Agriculture, Ecosystems and Environment, v. 133, p. 23-31, 2009. http://dx.doi.org/10.1016/j.agee.2009.04.016

MARTINS, F. B.; PEREIRA, R. A. A.; PINHEIRO, M. V. M.; ABREU, M. C. Desenvolvimento foliar em duas cultivares de oliveira estimado por duas categorias de modelos. Revista Brasileira de Meteorologia, v. 29, n. 4, p. 505514, 2014. http://dx.doi.org/10.1590/0102-778620140020

MAULIÓN, E.; VALENTINI, G. H.; KOVALEVSKI, L.; PRUNELLO, M.; MONTIA, L. L.; DAORDENB, M. E.; QUAGLINOC, M.; CERVIGNI, G. D. L. Comparison of methods for estimation of chilling and heat requirements of nectarine and peach genotypes for flowering. Scientia Horticulturae, v. 177, p. 112-117, 2014. http://dx.doi.org/10.1016/j.scienta.2014.07.042

MORAIS, H.; CARBONIERI, J. Horas e unidades de frio em pomares de maçã com diferentes microclimas. Revista Brasileira de Fruticultura, v. 37, p. 001012, 2015. http://dx.doi.org/10.1590/0100-2945-005/14

PEDRO JR., M. J.; ORTOLANI, A. A.; RIGITANO O.; ALFONSI, R. R.; PINTO, H. S.; BRUNINI, O. Estimativa de Horas de Frio abaixo de $7^{\circ} \mathrm{C}$ e de $13^{\circ} \mathrm{C}$ para regionalização da fruticultura de clima temperado no estado do São Paulo. Bragantia, v. 38 , n. 13, p. 123-130, 1979. http://dx.doi.org/10.1590/S000687051979000100013

POLA, A. C.; ANGELOCCI, L. R. Avaliação de modelos de estimativa do número diário de "horas de frio" para o estado de Santa Catarina. Revista Brasileira de Agrometeorologia, v. 1, p. 105-116, 1993. Disponível em: <http://www.sbagro.org.br/revistas/volumes_completos_do_1\%C2\%BA_ao_7\% C2\%BA_volume.pdf>. Acesso em: 07/05/2017.

RICHARDSON, E. A.; SEELEY, S. D.; WALKER, D. R. A model for estimating the completion of rest for Redhaven and Elberta peach trees. HortScience, v. 9, p. 331-332, 1974.

SILVA, E. M. S.; GUETTER, A. K. Mudanças climáticas regionais observadas no estado do Paraná. Terra Livre, v. I, n. 20, p. 111-126, 2003.

TANASIJEVIC, L.; TODOROVIC, M.; PEREIRA, L. S.; PIZZIGALLI, C.; LBIONELLO, $P$. Impacts of climate change on olive crop evapotranspiration and irrigation requirements in the Mediterranean region. Agricultural Water Management, v.144, p.54-68, 2014. https://doi.org/10.1016/j.agwat.2014.05.019

TORRES, R. R.; MARENGO, J. A. Climate change hotspots over South America: from CMIP3 to CMIP5 multi-model datasets. Theoretical and Applied Climatology, v. 117, n. 3, p. 579-587, 2014. https://doi.org/10.1007/s00704013-1030-x

WORLD METEOROLOGICAL ORGANIZATION (WMO). Guide to meteorological instruments and methods of observation. Geneva: WMO, 2008. Disponível em: <https://www.wmo.int/pages/prog/gcos/documents/gruanmanuals/CIMO/CIMO _Guide-7th_Edition-2008.pdf>. Acesso em: 12/05/2017. 
WREGE, M. S.; HERTER, F. G.; FRITZSONS, E. Regiões com similaridade de horas de frio no outono-inverno no sul do Brasil. Revista Brasileira de Climatologia, 18 ,

p. 108-118, 2016. http://dx.doi.org/10.5380/abclima.v18i0.43087 